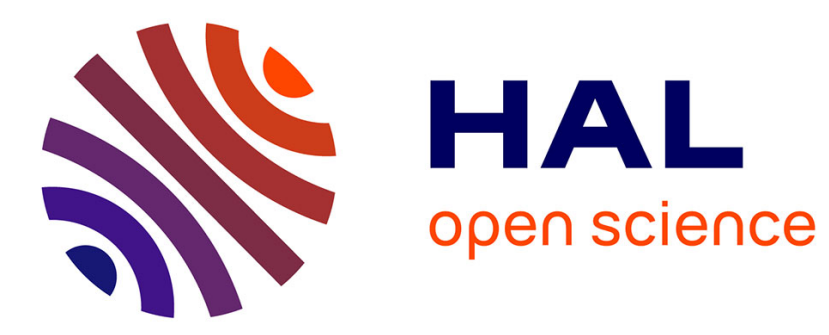

\title{
Interaction entre dislocations et défauts ponctuels créés par bombardement électronique dans l'argent
}

\author{
Pascal Girard, C. Minier
}

\section{To cite this version:}

Pascal Girard, C. Minier. Interaction entre dislocations et défauts ponctuels créés par bombardement électronique dans l'argent. Journal de Physique, 1978, 39 (9), pp.981-991. 10.1051/jphys:01978003909098100 . jpa-00208840

\section{HAL Id: jpa-00208840 https://hal.science/jpa-00208840}

Submitted on 1 Jan 1978

HAL is a multi-disciplinary open access archive for the deposit and dissemination of scientific research documents, whether they are published or not. The documents may come from teaching and research institutions in France or abroad, or from public or private research centers.
L'archive ouverte pluridisciplinaire HAL, est destinée au dépôt et à la diffusion de documents scientifiques de niveau recherche, publiés ou non, émanant des établissements d'enseignement et de recherche français ou étrangers, des laboratoires publics ou privés. 


\title{
INTERACTION ENTRE DISLOCATIONS ET DÉFAUTS PONCTUELS CRÉÉS PAR BOMBARDEMENT ÉLECTRONIQUE DANS L'ARGENT
}

\author{
P. GIRARD et C. MINIER \\ Centre d'Etudes Nucléaires de Grenoble, Département de Recherche Fondamentale, \\ Section de Physique du Solide, 85 X, 38041 Grenoble Cedex, France
}

(Reçu le 6 avril 1978, accepté le 31 mai 1978)

\begin{abstract}
Résumé. - Le module élastique et le frottement intérieur de l'argent bombardé par des électrons à des températures comprises entre $9 \mathrm{~K}$ et $300 \mathrm{~K}$ ont été étudiés. Le premier stade d'ancrage des dislocations qui apparaît entre $25 \mathrm{~K}$ et $50 \mathrm{~K}$ est attribué à la migration de l'interstitiel libre à longue distance.

D'autre part, lors d'irradiations à des températures au moins égales à $80 \mathrm{~K}$, le peaking effect a été observé et ses caractéristiques sont étudiées en fonction de la température d'irradiation, de la déformation de mesure, du flux instantané de particules incidentes et de la nature des échantillons. Ces résultats sont ensuite confrontés aux diverses théories qui tentent d'expliquer le peaking effect.

Abstract. - The elastic modulus and internal friction of silver irradiated by electrons in the temperature range 9-300 $\mathrm{K}$ have been studied. The first pinning stage for the dislocations appears between $25-50 \mathrm{~K}$ and is attributed to the long range migration of free interstitials.

Following irradiations at temperatures greater than or equal to $80 \mathrm{~K}$, the peaking effect has been observed and its characteristics studied as a function of the temperature of irradiation, strain amplitude, instantaneous flux of electrons and the purity of the samples. The results are critically compared with the various theories of the peaking effect.
\end{abstract}

1. Introduction. - On sait depuis un certain nombre d'années $[1,2]$ que les défauts ponctuels créés par irradiation, interstitiels et lacunes, interagissent avec les dislocations et modifient leur mouvement sous l'action d'une contrainte thermomécanique. Les mécanismes d'interaction qui entrent en jeu sont très nombreux, mais on peut cependant éliminer certains d'entre eux en se plaçant dans des conditions expérimentales particulières.

Dans le cuivre, nous avons montré [3] que si la déformation mécanique est très faible, les défauts ponctuels peuvent jouer le rôle de points d'ancrage immobiles et le mouvement de la dislocation est alors bien décrit par la théorie de Koehler [4] et GranatoLücke [5] qui l'assimile au mouvement d'une corde vibrante. Si la température est inférieure à celle du pic de Bordoni c'est alors plutôt le déplacement des décrochements géométriques qui contrôle le mouvement de la dislocation [6], mais formellement, les équations qui donnent le frottement intérieur et le défaut de module associés à ce mécanisme ne diffèrent des expressions de la théorie de GranatoLücke que par les valeurs de certaines constantes [7]. Dans ces conditions expérimentales, les mesures de frottement intérieur et de module élastique permettent de déduire le nombre de points d'ancrage présents sur un segment libre de dislocation avec une très grande sensibilité et sont donc très utiles pour l'étude des phénomènes de création, de migration et d'annihilation de ces défauts.

Dans l'argent, une étude de l'ancrage des dislocations par des défauts créés par rayonnement $\gamma$ a été réalisée au voisinage de la température ambiante [8]; elle présente également des résultats préliminaires pour les basses températures qui semblent indiquer que des défauts sont mobiles dans le cristal et arrivent sur les dislocations pour des températures de l'ordre de 65 à $90 \mathrm{~K}$. Or la migration de l'interstitiel libre dans l'argent qui est associée au stade $\mathrm{I}_{\mathrm{E}}$ de restauration de la résistivité après irradiation s'effectue entre 30 et $40 \mathrm{~K}[9,10]$. Nous avons réalisé des expériences pour clarifier ce point particulier que nous présentons dans le paragraphe 3.3.

Dans la partie suivante (3.4), nous avons voulu déterminer si l'effet anormal de variation du frottement intérieur observé par Simpson et Sosin au cours d'une irradiation dans le cuivre [11] et baptisé peaking effect existe dans l'argent. Les conditions d'existence de cet 
effet dans le cas du cuivre sont controversées [3, 12, 13], et il n'a pas été observé dans celui de l'argent par Van Konynenburg [8]; plusieurs théories tentent de l'expliquer $([11,14,15]$ et Mercier à paraître), et nous désirons les tester dans l'argent.

2. Techniques expérimentales. -2.1 PRÉPARATION DES ÉCHANTILLONS. - Les échantillons E1 et E2 sont des lames qui ont été préparées à partir de feuilles d'argent Purcla de pureté nominale 99,999\% de 0,2 mm d'épaisseur. L'échantillon E3 provient quant à lui d'une feuille d'argent Cominco de pureté 99,999 $9 \%$ et de même épaisseur.

La mise en forme des échantillons s'effectue par matriçage et ceux-ci sont ensuite soumis à un nettoyage chimique superficiel à l'acide nitrique suivi de nombreux rinçages, puis à un recuit sous un vide de $10^{-8}$ torr à $900 \mathrm{~K}$ pendant 4 heures. Un recuit in situ est effectué à $700 \mathrm{~K}$ après montage dans le cryostat afin d'éliminer un éventuel écrouissage accidentel.

2.2 DisPositIF EXPÉRIMENTAL. - Les échantillons sont soumis à un bombardement électronique d'énergie 1,5 MeV grâce à un accélérateur Van de Graaff. Le cryostat de mesure est associé à un cryogénérateur à hélium qui permet son utilisation dans une gamme de température étendue $(4 \mathrm{~K}-700 \mathrm{~K})$. Des recuits in situ à haute température peuvent ainsi être effectués afin d'effacer les défauts créés par l'irradiation et de retrouver les conditions initiales.

L'ensemble de ces techniques d'irradiation et de mesure à basse température a été décrit précédemment par Soulié et al. [16]. Les variations du module d'élasticité $M$ sont déterminées indirectement par celles de la fréquence $f$ de vibration du mode fondamental de flexion de la lame par la relation :

$$
\frac{\Delta M}{M}=2 \frac{\Delta f}{f} .
$$

Le frottement intérieur $Q^{-1}$ s'évalue en mesurant la tension d'excitation électrostatique appliquée à l'échantillon pour entretenir l'oscillation à amplitude constante. Une décroissance libre permet un étalonnage constant de cette mesure.

3. Résultats expérimentaux. - 3.1 SPECTRE DE FROTTEMENT INTÉRIEUR AVANT IRRADIATION. - La figure 1 montre la variation du frottement intérieur de l'échantillon E1 d'argent recuit à $900 \mathrm{~K}$, sous un vide de $10^{-8}$ torr, puis recuit in situ à $700 \mathrm{~K}$ après montage dans le cryostat, en fonction de la température. Sa fréquence de vibration est de l'ordre de $260 \mathrm{~Hz}$.

Nous observons un large pic culminant entre 65 et $70 \mathrm{~K}$ qui présente un épaulement du côté basse température nettement confirmé sur d'autres échan-

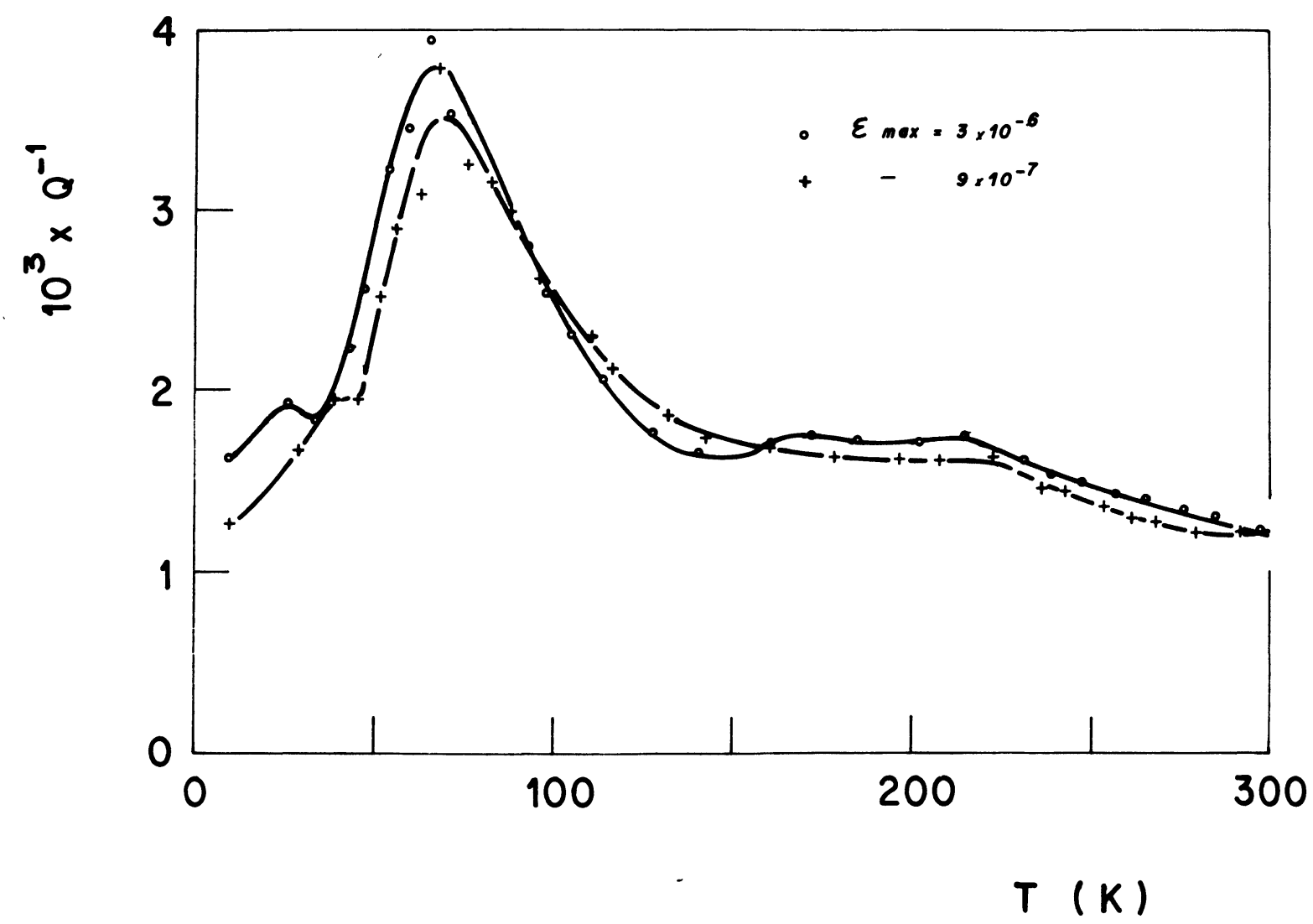

Fig. 1. - Frottement intérieur de l'échantillon E1 recuit à $700 \mathrm{~K}$ en fonction de la température pour deux déformations différentes $(f=260 \mathrm{~Hz})$.

[Internal friction for specimen $\mathrm{E} 1$ annealed at $700 \mathrm{~K}$ as a function of temperature for two different deformations.] 


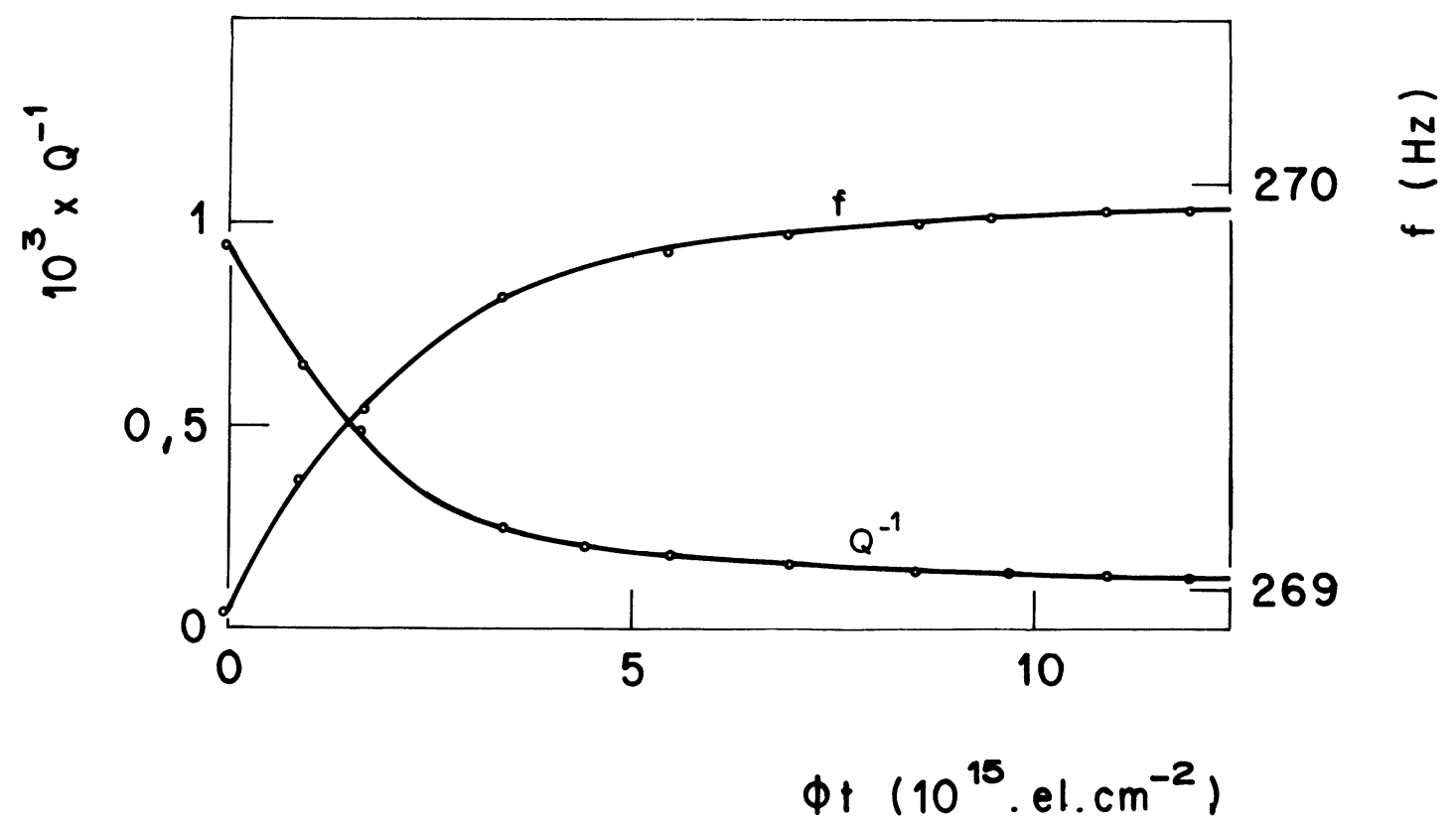

FIG. 2. - Fréquence de résonance et frottement intérieur de l'échantillon E1 au cours d'une irradiation à $20 \mathrm{~K}$.

[Resonance frequency and internal friction for specimen E1 during an irradiation at $20 \mathrm{~K}$.]

tillons, et des pics larges et mal distingués au-dessus de $150 \mathrm{~K}$. Le frottement intérieur augmente avec la déformation mécanique appliquée, sauf entre 100 et $150 \mathrm{~K}$ où il en est à peu près indépendant.

3.2 VARIATION DU MODULE ÉLASTIQUE ET DU FROTTEMENT INTÉRIEUR LORS D'UNE IRRADIATION A BASSE TEMPÉRATURE. - La figure 2 montre les variations de la fréquence de résonance et du frottement intérieur de l'échantillon $E 1$ au cours d'un bombardement par des électrons de $1,5 \mathrm{MeV}$ à $9,5 \mathrm{~K}$.

3.3 EVOLUTION DU FROTTEMENT INTÉRIEUR ET DU MODULE ÉLASTIQUE D'UN ÉCHANTILLON IRRADIÉ A BASSE TEMPÉRATURE APRÈS DES RECUITS ISOCHRONES A TEMPÉRATURE CROISSANTE. - La figure 3 montre les

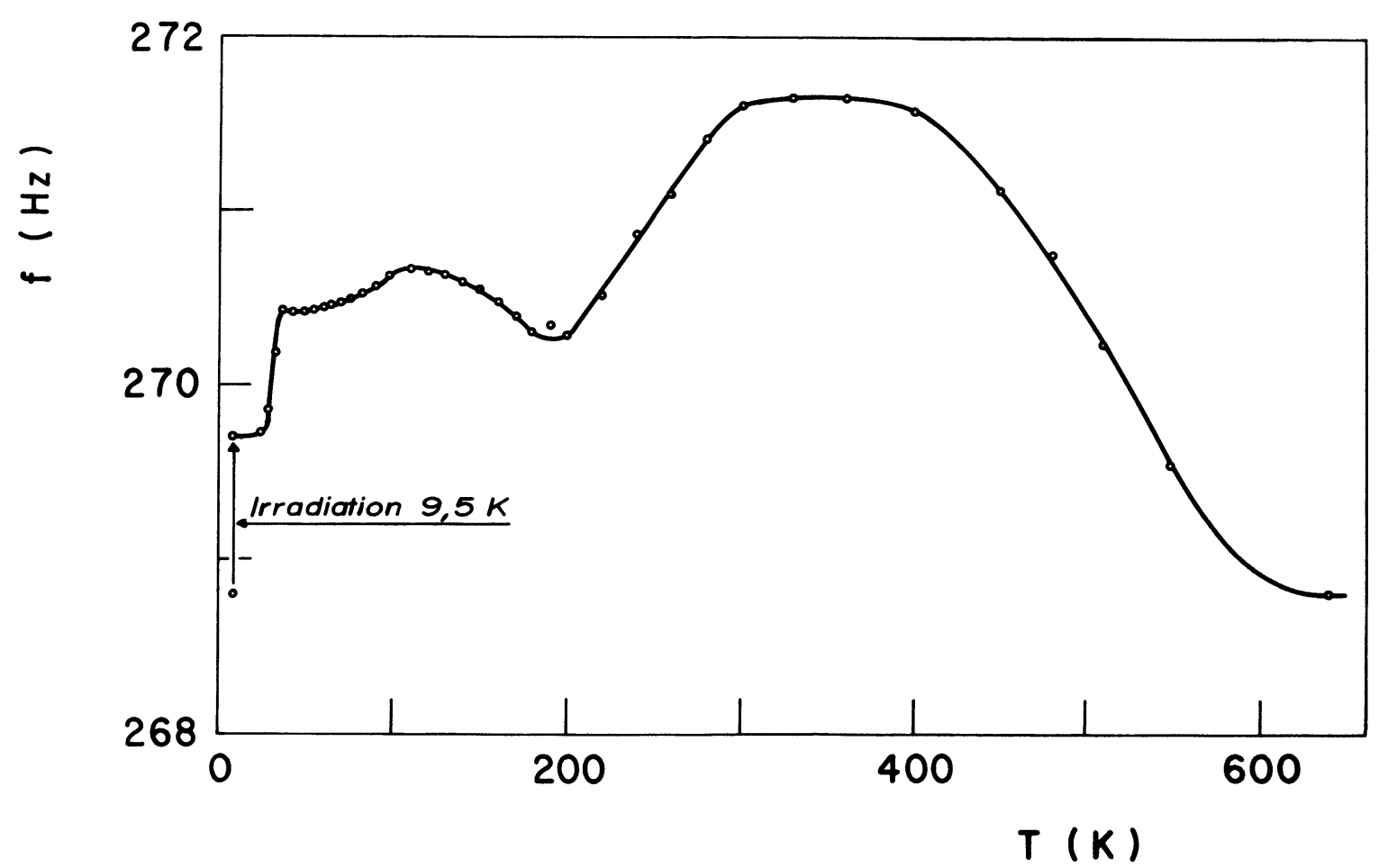

Fig. 3. - Fréquence de résonance mesurée à $9,5 \mathrm{~K}$ de l'échantillon $\mathrm{E} 1$ après irradiation à cette même température et des recuits isochrones à température $T$ croissante.

[Resonance frequency measured at $9.5 \mathrm{~K}$ for specimen $\mathrm{E} 1$ after an irradiation at the same temperature and equal time anneals at an increasing temperature $T$.] 
variations de la fréquence de résonance de l'échantillon E1 après un bombardement électronique d'énergie $1,5 \mathrm{MeV}$ à $9,5 \mathrm{~K}$, le flux total reçu étant égal à $7,5 \times 10^{17} \mathrm{el} / \mathrm{cm}^{2}$. Les mesures sont effectuées à la température de référence de $9,5 \mathrm{~K}$ après des recuits isochrones successifs de 10 minutes à des températures croissantes.

La figure 4 montre de la même façon les variations de la fréquence de résonance et du frottement intérieur dans le domaine des températures inférieures à $65 \mathrm{~K}$ pour l'échantillon E2, celui-ci ayant subi un bombardement électronique à $9,5 \mathrm{~K}$ de même énergie, le flux total reçu est ici de $7,5 \times 10^{15} \mathrm{el} / \mathrm{cm}^{2}$.

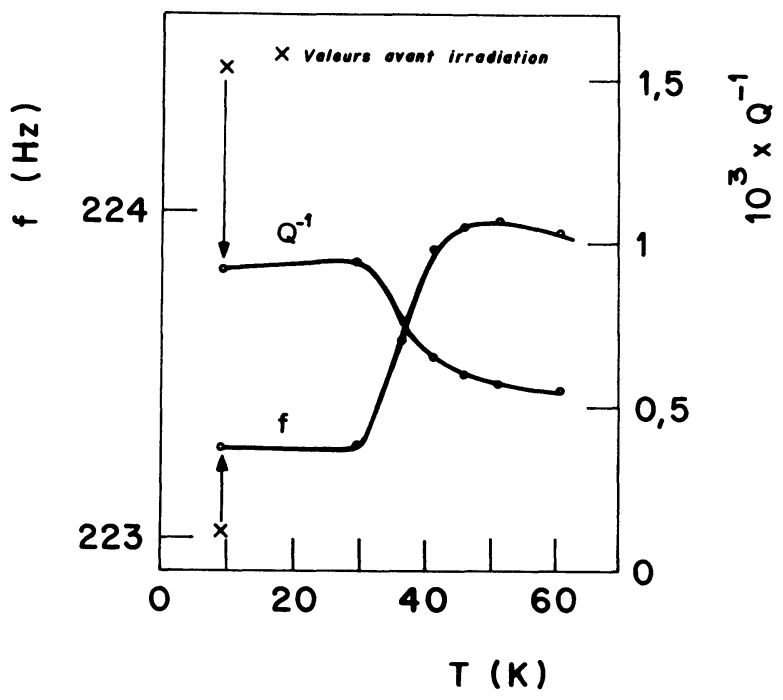

FIG. 4. - Fréquence de résonance et frottement intérieur mesurés à $9,5 \mathrm{~K}$ de l'échantillon $\mathrm{E} 2$ après irradiation à cette même température et recuits isochrones à température $T$ croissante jusqu'à $60 \mathrm{~K}$.

[Resonance frequency and internal friction measured at $9.5 \mathrm{~K}$ for specimen E2 after an irradiation at the same temperature and equal time anneals at an increasing temperature $T$ to $60 \mathrm{~K}$.]

La figure 5 est l'analogue de la figure 4 pour l'échantillon E3, le flux total d'électron reçu étant ici de $7,2 \times 10^{15} \mathrm{el} / \mathrm{cm}^{2}$.

3.4 EVOLUTION DU FROTTEMENT INTÉRIEUR ET DU MODULE ÉLASTIQUE LORS D'IRRADIATIONS A TEMPÉRATURES SUPÉRIEURES A CELLES DU STADE I. - Les expériences qui suivent ont toutes été réalisées sur l'échantillon E2. Après chacune des irradiations, un recuit effectué dans le cryostat d'irradiation à une température de $650 \mathrm{~K}$, sous un vide de $10^{-6}$ torr et pendant 1 heure permet de retrouver les valeurs initiales de la fréquence de résonance et du frottement intérieur.

La figure 6 montre l'évolution au cours du temps de la fréquence de résonance et du frottement intérieur de l'échantillon soumis à un bombardement électronique de flux instantanés $\Phi=8,3 \times 10^{11} \mathrm{el} / \mathrm{cm}^{2}$.s à une température de $170 \mathrm{~K}$. On peut constater que la fréquence de résonance croît de façon monotone alors que le frottement intérieur passe par un maximum

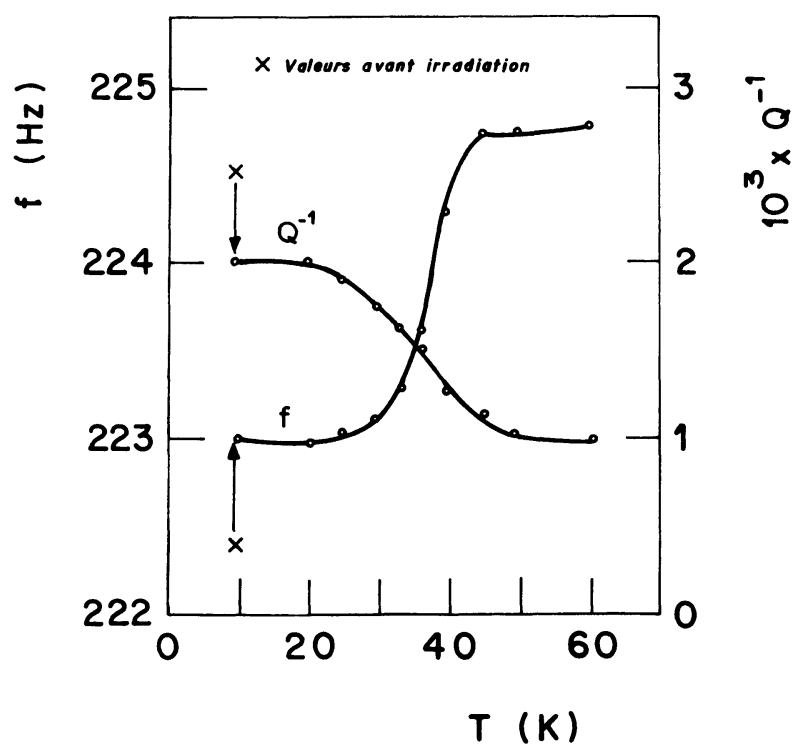

FIG. 5. - Fréquence de résonance et frottement intérieur mesurés à $9,5 \mathrm{~K}$ de l'échantillon $\mathrm{E} 3$ après irradiation à cette même température et recuits isochrones à température $T$ croissante jusqu'à $60 \mathrm{~K}$.

[Resonance frequency and internal friction measured at $9.5 \mathrm{~K}$ for specimen E3 after an irradiation at the same temperature and equal time anneals at an increasing temperature $T$ to $60 \mathrm{~K}$.]

qui est caractéristique du peaking effect. Nous avons entrepris une série d'expériences pour caractériser l'évolution de ce phénomène avec l'amplitude de la déformation de mesure, le flux instantané des particules incidentes, la température d'irradiation et la nature de l'échantillon.

a) Influence de l'amplitude de la déformation de mesure. - La figure 7 montre les variations au cours du temps du frottement intérieur normalisé à l'origine, au cours d'une irradiation de flux instantané : $\Phi=2,8 \times 10^{11} \mathrm{el} / \mathrm{cm}^{2} . s$ pour des déformations maximum variant entre $1,2 \times 10^{-6}$ et $3,6 \times 10^{-6}$. On observe que le peaking effect augmente nettement avec l'amplitude de la déformation. Le maximum se situe d'autant plus tard que la déformation est importante.

b) Influence du flux instantané de particules incidentes. - La figure 8 montre la variation du frottement intérieur et du défaut de module normalisés, avec le flux intégré d'électrons incidents au cours de trois irradiations réalisées à $170 \mathrm{~K}$ avec des flux instantanés variant dans les proportions 1,3 , 9, la déformation de mesure étant égale à $\varepsilon_{\max }=2,5 \times 10^{-6}$. Sachant que chacune de ces courbes correspond à une irradiation différente, ces expériences ayant été faites à plusieurs jours d'intervalle, on peut considérer qu'il n'y a pas de différences signicatives entre elles.

c) Influence de la températurá d'irradiation. - La figure 9 montre la variation du frottement intérieur normalisé mesuré pour une déformation maximum de $2,6 \times 10^{-6}$ au cours de bombardements électroniques de flux instantané $\Phi=8,3 \times 10^{11} \mathrm{el} / \mathrm{cm}^{2} . \mathrm{s}$ 


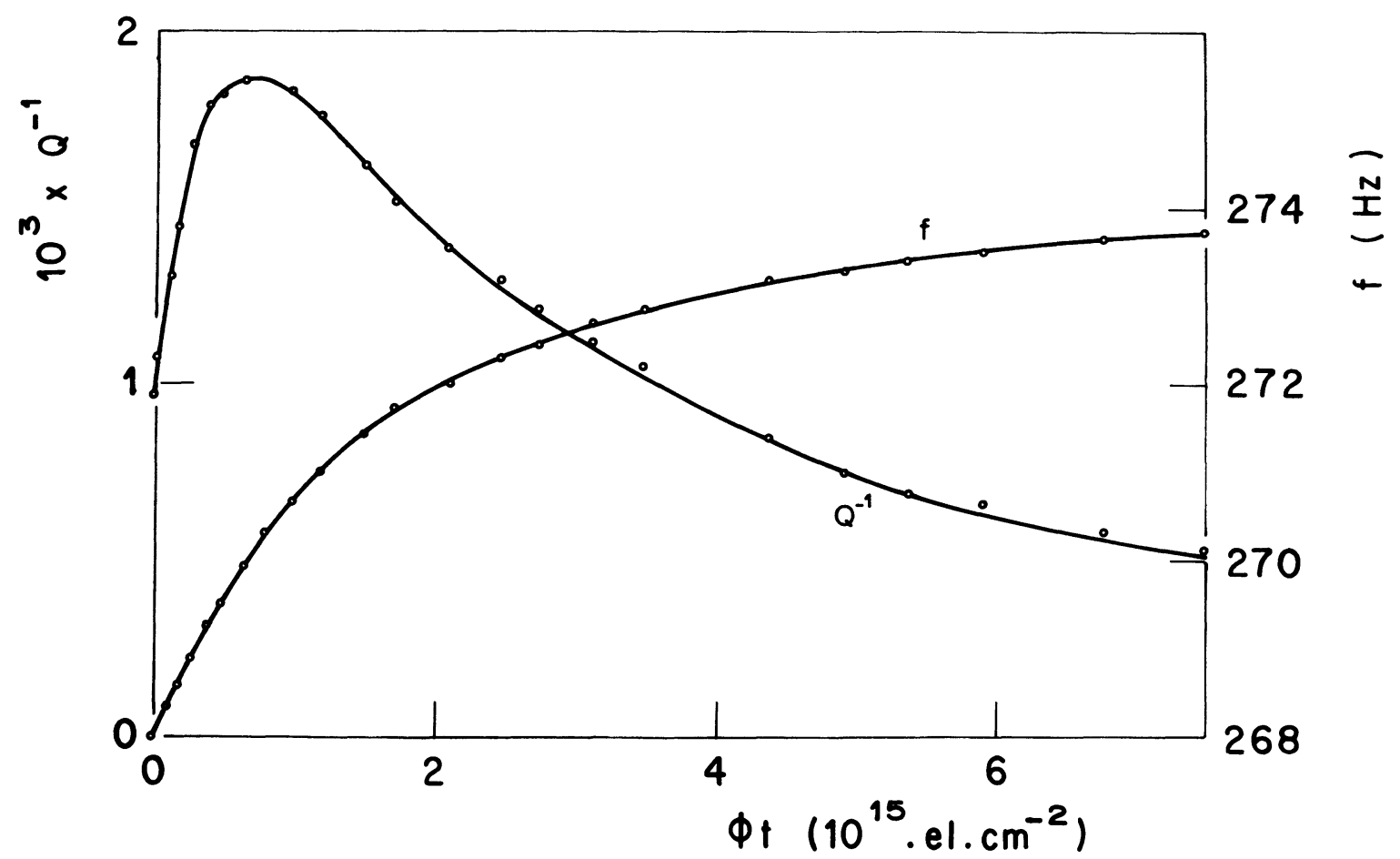

Fig. 6. - Fréquence de résonance et frottement intérieur de l'échantillon E2 au cours d'une irradiation à $170 \mathrm{~K}$ de flux instantané $\Phi=8,3 \times 10^{11} \mathrm{el} / \mathrm{cm}^{2} . \mathrm{s}$.

[Resonance frequency and internal friction for specimen E2 during an irradiation at $170 \mathrm{~K}$ in an instantaneous flux $\Phi=8.3 \times 10^{11} \mathrm{el} / \mathrm{cm}^{2}$.s.]

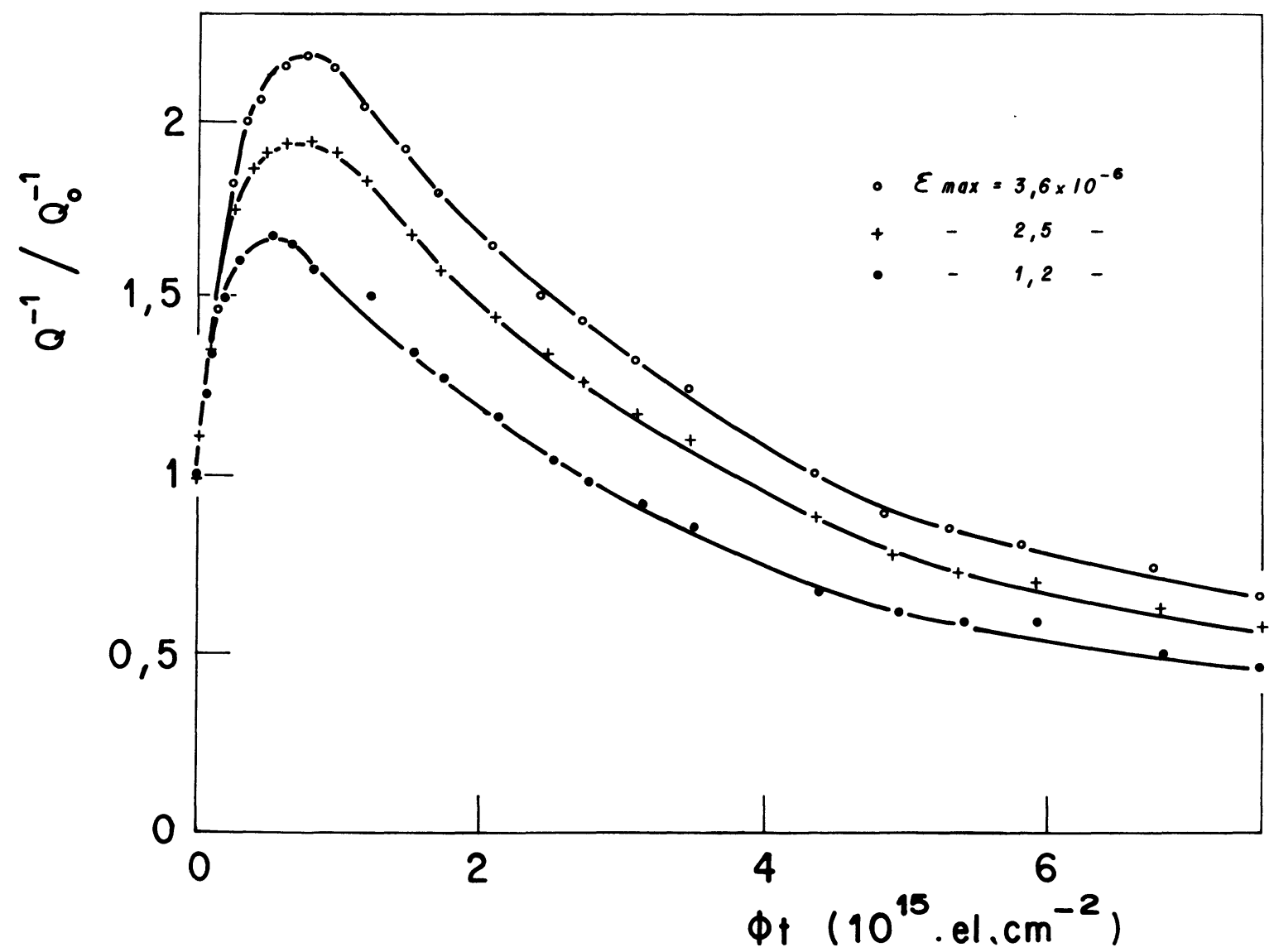

Fig. 7. - Frottement intérieur normalisé à l'origine de l'échantillon $\mathrm{E} 2$ au cours d'une irradiation à $170 \mathrm{~K}$ pour 3 déformations différentes $\left(\Phi=2,8 \times 10^{11} \mathrm{el} / \mathrm{cm}^{2} . \mathrm{s}\right)$.

[Internal friction normalized at time zero for specimen $\mathrm{E} 2$ during an irradiation at $170 \mathrm{~K}$ for 3 different deformations

$$
\left.\left(\Phi=2.8 \times 10^{11} \mathrm{el} / \mathrm{cm}^{2} . \mathrm{s}\right) \cdot\right]
$$




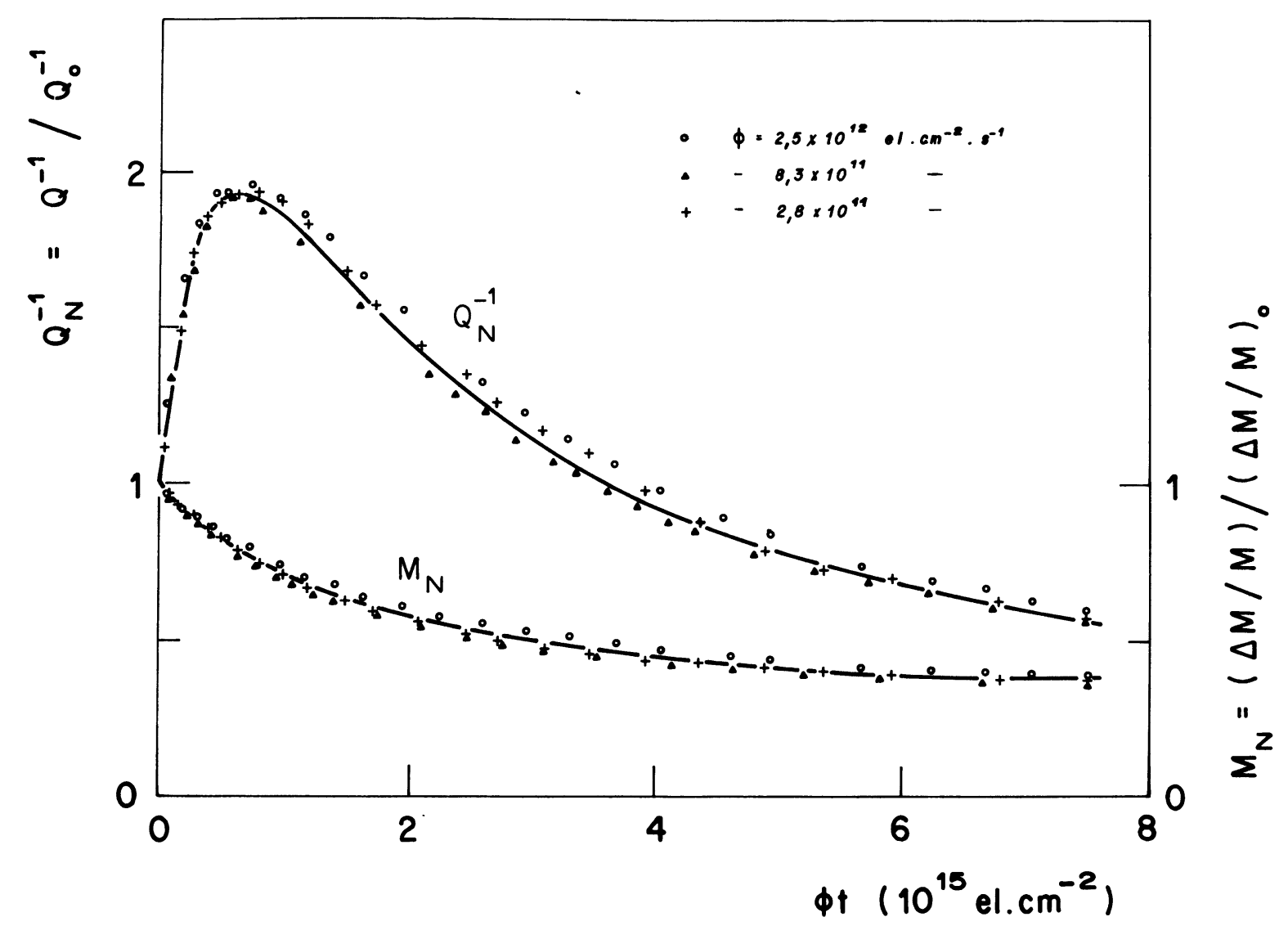

Fig. 8. - Frottement intérieur et défaut de module normalisés à l'origine de l'échantillon E2 en fonction du flux intégré $\Phi t$ au cours de 3 irradiations à $170 \mathrm{~K}$ de flux instantanés différents.

[Internal friction and modulus difference normalized at time zero for specimen E2 as a function of integrated flux $\Phi t$ during 3 irradiations at $170 \mathrm{~K}$ of different instantaneous fluxes.]

effectués à différentes températures comprises entre $50 \mathrm{~K}$ et $300 \mathrm{~K}$. On remarque que le peaking effect est absent à $50 \mathrm{~K}$, qu'il s'amorce à $80 \mathrm{~K}$, puisque la valeur de son maximum augmente avec la température. On peut noter également une variation de la valeur du flux intégré $(\Phi t)_{\mathrm{M}}$ correspondant au maximum du frottement interne : $(\Phi t)_{\mathrm{M}}$ augmente avec la température jusqu'à environ $190 \mathrm{~K}$ et diminue ensuite jusqu'à $300 \mathrm{~K}$ comme le montre la figure 10 .

d) Influence de la nature de l'échantillon. - Des expériences analogues ont été réalisées sur l'échantillon E3 et la figure 11 montre l'évolution du frottement intérieur normalisé à l'origine au cours d'un bombardement réalisé à $200 \mathrm{~K}$ par des électrons de flux instantané $\Phi=5,2 \times 10^{11} \mathrm{el} / \mathrm{cm}^{2}$.s. La déformation de mesure est de $2 \times 10^{-6}$. On constate ici que pour une valeur initiale du frottement intérieur de $6 \times 10^{-4}$ la valeur maximum atteinte est presque trois fois plus élevée que celle-ci. Pour une irradiation réalisée dans des conditions sensiblement équivalentes sur l'échantillon E2 et pour une valeur initiale du frottement intérieur de $10^{-3}$, la valeur maximum atteinte n'était qu'environ deux fois plus élevée que la valeur de départ.
De plus on constate ici que le flux intégré d'électrons $(\Phi t)_{\mathrm{M}}$ correspondant au maximum du peaking effect est sensiblement moins élevé dans le cas de cet échantillon que dans celui de l'échantillon E2, soit

$$
\begin{array}{ll}
(\Phi t)_{\mathrm{M}} \simeq 0,2 \times 10^{15} \mathrm{el} / \mathrm{cm}^{2} & \text { échantillon } \mathrm{E} 3 \\
(\Phi t)_{\mathrm{M}} \simeq 0,6 \times 10^{15} \mathrm{el} / \mathrm{cm}^{2} & \text { échantillon E2 } .
\end{array}
$$

4. Discussion. - 4.1 IDentification du STADE DE MIGRATION DES INTERSTITIELS LIBRES DANS L'ARGENT. - Les figures 3, 4 et 5 montrent que dans un échantillon d'argent irradié à une température inférieure à $20 \mathrm{~K}$, on observe au cours de recuits isochrones une augmentation nette de fréquence accompagnée d'une baisse de frottement intérieur entre $20 \mathrm{~K}$ et $50 \mathrm{~K}$. Ces variations sont caractéristiques d'un ancrage des dislocations par un défaut qui a migré dans le réseau cristallin à ces températures, et que nous identifions à l'auto-interstitiel. Remarquons aussi que pour une même irradiation l'échantillon E3, le plus pur, présente une variation de module et de frottement intérieur au cours du stade I plus importante que pour l'échantillon E2. 


\section{$\dagger(\min )$}

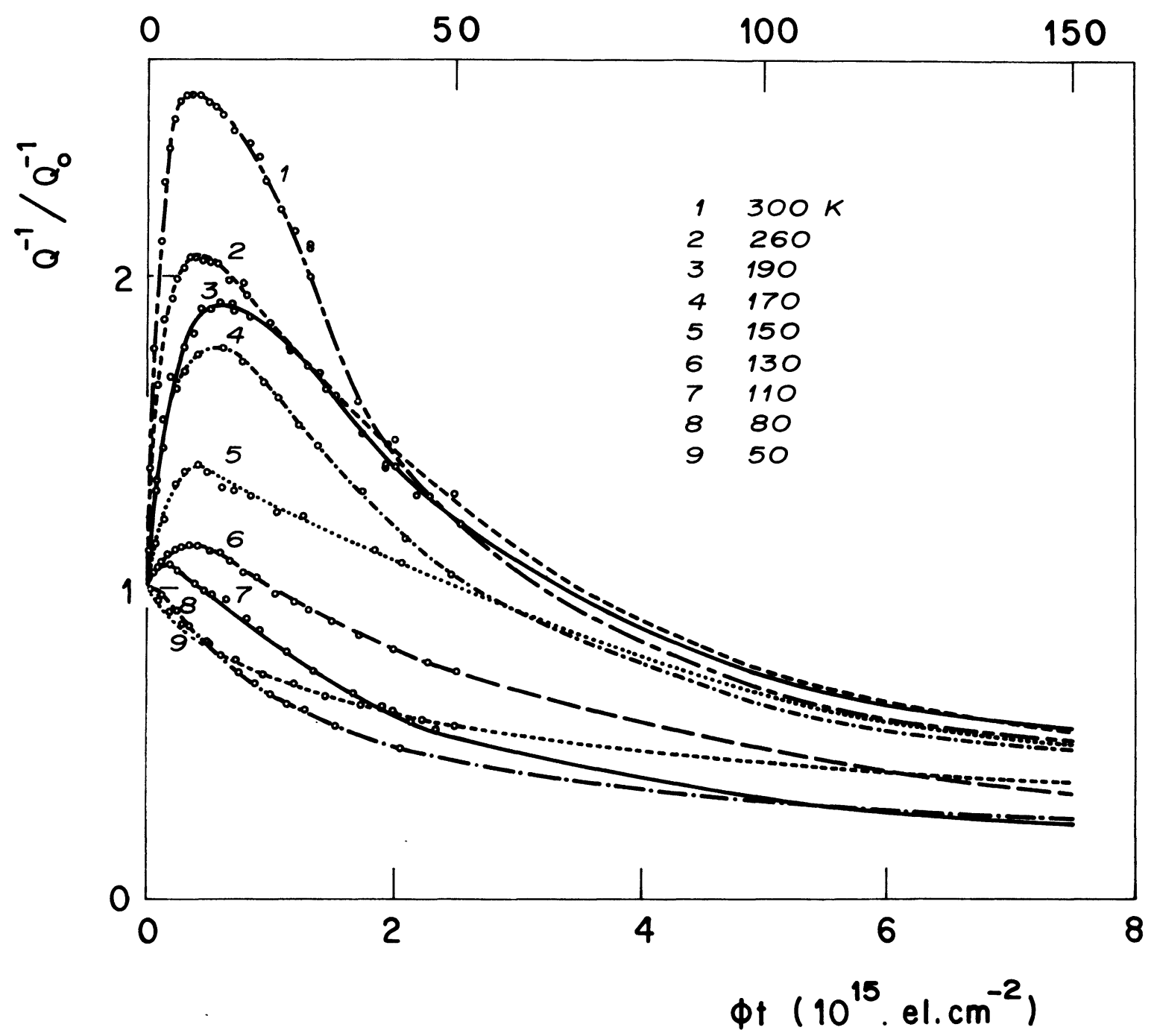

Fig. 9. - Frottement intérieur normalisé à l'origine de l'échantillon E2 au cours d'irradiations à températures différentes

$$
\left(\Phi=8,3 \times 10^{11} \mathrm{el} / \mathrm{cm}^{2} . \mathrm{s}\right) .
$$

[Internal friction normalized at time zero for specimen E2 during irradiation at different temperatures $\left(\Phi=8.3 \times 10^{11} \mathrm{el} / \mathrm{cm}^{2} . \mathrm{s}\right)$.]

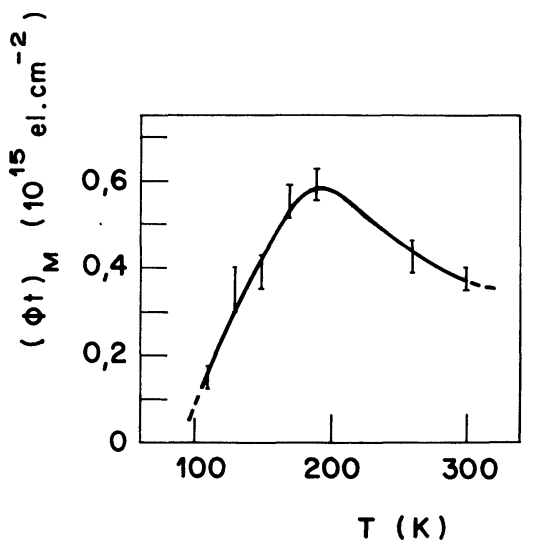

Fig. 10. - Flux intégré d'électrons $(\Phi t)_{\mathrm{M}}$ correspondant au maximum du peaking effect pour différentes températures d'irradiation.

[Integrated flux of electrons $(\Phi t)_{\mathrm{M}}$ corresponding to the maximum of the peaking effect for different temperatures of irradiation.]
Des mesures de résistivité électrique de l'argent bombardé par des électrons [9] montrent la présence de plusieurs sous-stades de revenu de "la résistivité à basse température. Les sous-stades $I_{B}$ et $I_{C}$ situés en dessous de $20 \mathrm{~K}$ sont attribués à l'annihilation de paires de Frenkel proches; un important stade $I_{D}$ présentant un épaulement $I_{E}$ est situé entre $22 \mathrm{~K}$ et $38 \mathrm{~K} ; \mathrm{I}_{\mathrm{E}}$ est attribué à la migration libre de l'interstitiel qui va s'annihiler dans une lacune avec laquelle il n'est pas en interaction, mais contrairement au cas du cuivre irradié, il n'a été observé que de très faibles déplacements de la température du stade avec la concentration des paires de Frenkel $[9,10]$.

Le stade d'ancrage des dislocations que nous observons entre 25 et $50 \mathrm{~K}$ peut être associé comme les stades $I_{D}$ et $I_{E}$ à la migration de l'interstitiel libre. Il s'étend jusqu'à une température plus élevée car 


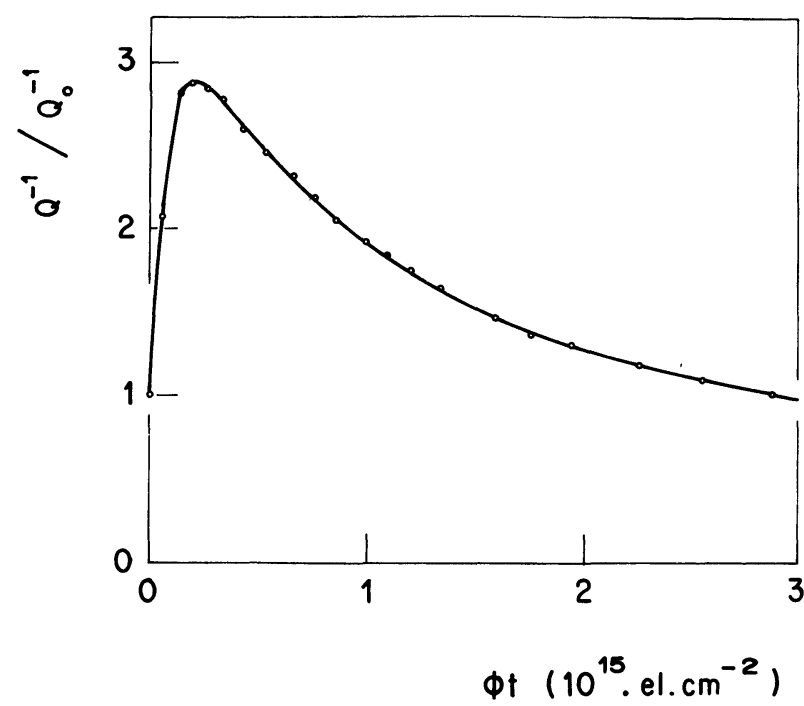

FIG. 11. - Frottement interne normalisé à l'origine de l'échantillon E3 au cours d'une irradiation à $200 \mathrm{~K}\left(\Phi=5,2 \times 10^{11} \mathrm{el} / \mathrm{cm}^{2} . \mathrm{s}\right)$

[Internal friction normalized at time zero for specimen E3 during an irradiation at $\left.200 \mathrm{~K}\left(\Phi=5.2 \times 10^{11} \mathrm{el} / \mathrm{cm}^{2} . \mathrm{s}\right).\right]$

certains interstitiels créés au sein du cristal doivent faire un nombre de sauts plus important pour arriver sur une dislocation et être observés par notre technique que le nombre de sauts moyen pour l'annihilation dans une lacune qui est responsable de la baisse de résistivité.

$\mathrm{Si}$ nous comparons maintenant plus en détail les zones de températures du stade d'ancrage des dislocations des trois échantillons étudiés (Figs 3, 4 et 5), nous observons que la température d'ancrage maximum est d'environ $32 \mathrm{~K}$ pour l'échantillon $\mathrm{E} 1$ qui a subi un bombardement de $7 \times 10^{17} \mathrm{el} / \mathrm{cm}^{2}$ et de $37 \mathrm{~K}$ pour les échantillons E2 et E3 qui ont chacun subi un bombardement de l'ordre de $7 \times 10^{15} \mathrm{el} / \mathrm{cm}^{2}$. Ce déplacement se comprend bien qualitativement car les interstitiels peuvent effectuer un nombre de sauts d'autant plus important sans se recombiner que la concentration des paires de Frenkel est faible; l'ordre de grandeur du rapport des nombres de sauts observés dans les deux types d'irradiation est de quelques centaines. Il est par contre exclu d'interpréter les phénomènes d'ancrage observés par Van Konynenburg [8], après bombardement $\gamma$, entre 65 et $90 \mathrm{~K}$ par le même mécanisme ; en effet, dans les irradiations qu'il a réalisées à très faibles doses et dans des monocristaux qui peuvent contenir un taux global d'impuretés de l'ordre de $10^{-4}$, ce sont alors les impuretés qui limitent le nombre de sauts effectués par les interstitiels qui y sont ensuite piégés. Le stade d'ancrage observé entre 65 et $90 \mathrm{~K}$ pourrait très bien correspondre à la dissociation de complexes interstitiel-impureté.

4.2 ETUDE DU peaking effect. - Les expériences que nous avons réalisées montrent que ce phénomène peut exister dans l'argent et elles précisent son comportement vis-à-vis de la température d'irradiation, du flux instantané de particules incidentes et de la déformation.

De nombreuses théories existent pour expliquer l'ancrage des dislocations à des températures moyennes. Thompson et al. [17] présentent une analyse valable pour le cuivre qui permet de calculer le nombre de défauts sur une dislocation en tenant compte d'une part des défauts créés dans le cristal et qui migrent vers les lignes de dislocation, et en faisant d'autre part intervenir des processus de diffusion des défauts le long des dislocations vers les points nodaux, de l'absorption et de la réémission des défauts vers les lignes en ces points, ainsi que de la réémission de défauts, situés sur les dislocations, vers le cristal ; chacun de ces processus est caractérisé par un temps de relaxation.

Les trois irradiations que nous avons réalisées dans l'argent à $170 \mathrm{~K}$ avec des flux instantanés variables et représentées sur la figure 8 montrent que le frottement intérieur et le module élastique donc le nombre des défauts sur une longueur donnée de dislocation ne dépendent que du flux intégré $\Phi t$, c'est donc que les temps de relaxation associés aux différents processus d'élimination des défauts sur les lignes sont longs devant la durée des expériences.

Le frottement intérieur associé aux dislocations a été interprété par Koehler [4] et Granato et Lücke [5] en considérant le mouvement d'une dislocation comparable à celui d'une corde vibrante. Le déplacement $y(x, t)$ d'un petit segment de la dislocation situé à une distance $x$ d'une extrémité fixe est donné par l'équation :

$$
A \frac{\partial^{2} y}{\partial t^{2}}+B \frac{\partial y}{\partial t}+K y=\sigma_{0} b \mathrm{e}^{i \omega t}
$$

$\sigma_{0}$ est l'amplitude de la contrainte sinusoïdale appliquée de pulsation $\omega, A$ la masse effective de la dislocation par unité de longueur; $B$ est un coefficient de frottement visqueux et $K$ est la constante de rappel qui est associée à la tension de ligne $C$ par la relation $K=\pi^{2} C / l^{2}$ où $l$ est la longueur libre de la dislocation entre deux points d'ancrage pour lesquels $y=0$.

Les premières mesures sous irradiation [1] montrant une diminution du frottement intérieur et une augmentation du module élastique ont été interprétées en supposant que l'arrivée de défauts ponctuels diminuait la longueur libre $l$ sans modifier $B$; c'est le mécanisme d'ancrage ferme qui ne peut pas expliquer l'observation $\mathrm{du}$ peaking effect.

Pour interpréter ce dernier phénomène, Simpson et Sosin [11] ont proposé au contraire que les défauts ponctuels présents sur les dislocations ne restent pas immobiles mais sont entraînés dans un mouvement latéral. Ils supposent que la constante de rappel $K$ reste inchangée, mais que le coefficient de viscosité $B$ augmente, en première approximation, linéairement avec le nombre de défauts présents sur la ligne. Ce modèle explique bien leurs résultats de frottement intérieur dans le cuivre, mais pas les variations du module élastique. 
Plus récemment, Granato [15] et Lenz et Lücke [13] ont montré qu'il était intéressant, pour tester les différents modèles, de déterminer expérimentalement les variations de $B$ et $K$ pendant l'irradiation. Ceci implique des mesures simultanées du frottement intérieur et du module élastique et la détermination des valeurs élastiques $Q_{\mathrm{e}}^{-1}$ et $M_{\mathrm{e}}$ correspondant à ces quantités, donc en l'absence de dislocations mobiles. Le défaut de module et le frottement intérieur dus aux dislocations sont alors :

$$
\frac{\Delta M}{M}=\frac{M_{\mathrm{e}}-M_{\mathrm{m}}}{M_{\mathrm{m}}} \text { et } \Delta Q^{-1}=Q_{\mathrm{m}}^{-1}-Q_{\mathrm{e}}^{-1}
$$

où l'indice $\mathrm{m}$ correspond aux grandeurs mesurées.

Si la densité de dislocations est $\lambda$, l'amplitude du vecteur de Burgers $b$, le calcul [15] montre que $B$ et $K$ sont donnés par les expressions suivantes

$$
\begin{aligned}
& B=\frac{\lambda M b^{2}}{\omega} \frac{\Delta Q^{-1}}{\left(\frac{\Delta M}{M}\right)^{2}+\left(\Delta Q^{-1}\right)^{2}}, \\
& K=\lambda M b^{2} \frac{\frac{\Delta M}{M}}{\left(\frac{\Delta M}{M}\right)^{2}+\left(\Delta Q^{-1}\right)^{2}} .
\end{aligned}
$$

Les valeurs élastiques $M_{\mathrm{e}}$ et $Q_{\mathrm{e}}^{-1}$ ont donc ici une grande importance qui n'a pas toujours été suffisamment prise en considération. Elles peuvent être obtenues en bloquant complètement le mouvement des dislocations en les ancrant par des défauts; nous obtenons cet effet par une irradiation à une température de $170 \mathrm{~K}$, la saturation étant obtenue pour un flux électronique total de l'ordre de $10^{18} \mathrm{el} / \mathrm{cm}^{2}$.

La figure 12 montre les variations de $B$ et $K$ normalisés aux valeurs initiales au cours d'une irradiation à $170 \mathrm{~K}$. On peut constater que $K$ ne reste pas constant et que $B$ varie également, ses variations n'étant pas linéaires par rapport au flux intégré de particules incidentes. Toute interprétation dans un modèle de corde vibrante devra donc prendre en compte une influence des défauts ponctuels à la fois sur la constante de rappel et sur le coefficient de viscosité.

Une nouvelle analyse de Simpson et Sosin [18] envisage maintenant ce genre de mécanisme en conservant le même formalisme que dans leur précédente interprétation, mais en laissant le coefficient $B$ prendre une partie imaginaire.

Seeger [14] a proposé une interprétation du peaking effect fondée sur un effet Snoek des auto-interstitiels près des dislocations. Le peaking effect doit donc exister dans une zone de température qui correspond en position et largeur à celle d'un pic de Snoek. Nos expériences vérifient bien la disparition prévue du phénomène lorsque, la température diminuant, le temps de relaxation devient nettement plus grand que la période de la contrainte appliquée ; le peaking effect que nous observons dans l'argent existe dans une plage de température très large et le temps caractéristique du sommet du pic varie assez peu avec la température comme le montre la figure 10 ; ces observations pourraient peut-être s'interpréter en tenant compte

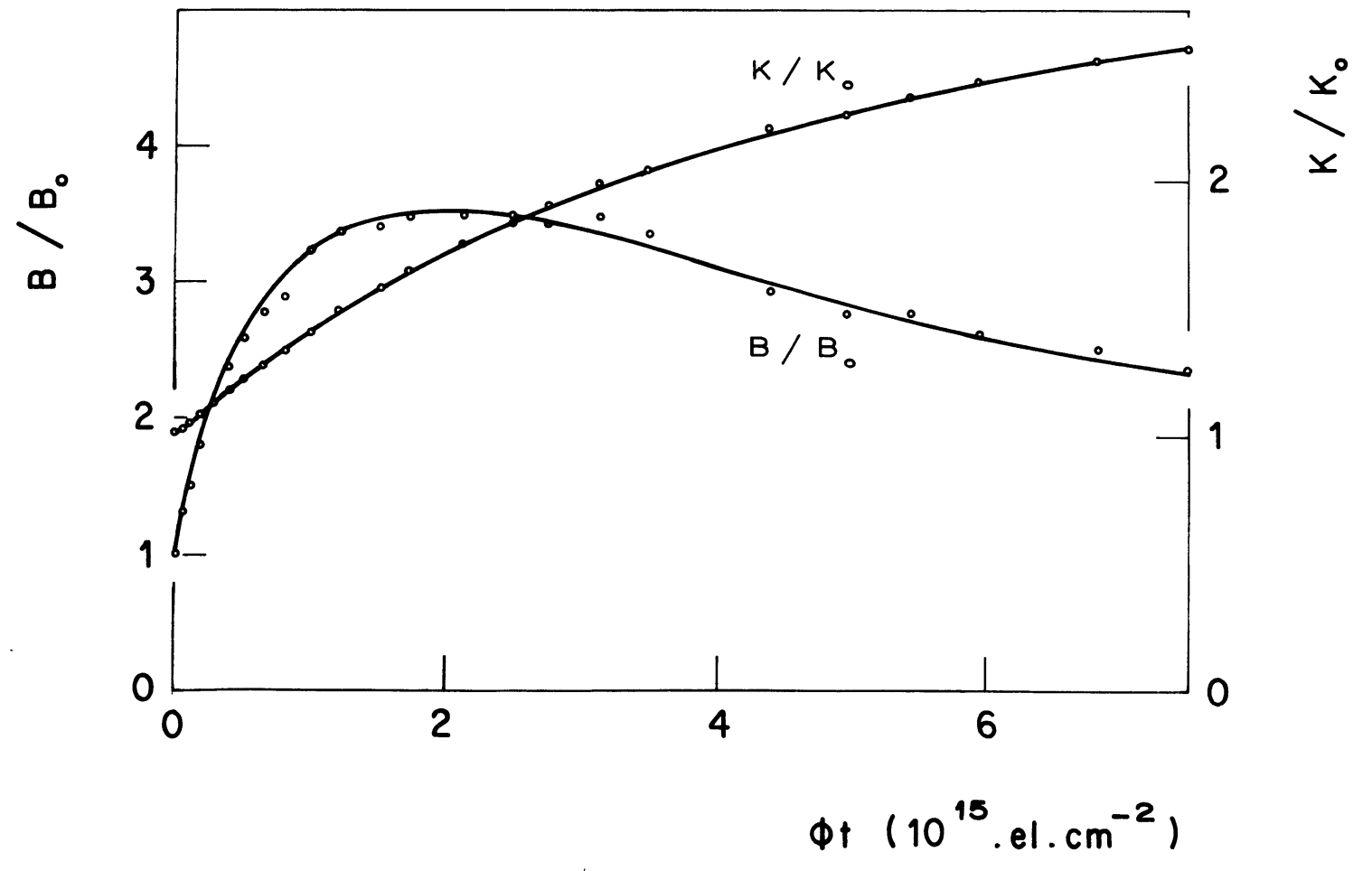

Fig. 12. - Valeurs normalisées à l'origine des coefficients $B$ et $K$ au cours d'une irradiation à $170 \mathrm{~K}\left(\Phi=8,3 \times 10^{11} \mathrm{el} / \mathrm{cm}^{2} . \mathrm{s}\right)$.

[Normalized values at time zero of the coefficients $B$ and $K$ during an irradiation at $\left.170 \mathrm{~K}\left(\Phi=8.3 \times 10^{11} \mathrm{el} / \mathrm{cm}^{2} \cdot \mathrm{s}\right) \cdot\right]$ 
dans la théorie d'un effet de l'amplitude de la déformation qui élargit et décale les pics de relaxation.

Il existe une interprétation plus récente du peaking effect qui lui donne une origine différente. Mercier (à paraître) considère que le mouvement des dislocations doit être décrit à basse température par celui des décrochements géométriques alors qu'au-dessus de la température du pic de Bordoni interviennent à la fois les décrochements géométriques et les doubles décrochements créés thermiquement; ces derniers n'apparaissent que pour des longueurs libres de dislocations supérieures à une valeur critique $l_{\mathrm{c}}$ donnée par la condition de Paré [19]

$$
\sigma_{\mathrm{i}} b l_{\mathrm{c}} a=2 W_{\mathrm{K}}
$$

$\sigma_{\mathrm{i}}$ est la contrainte interne, $b$ le vecteur de Burgers, $a$ le paramètre de la maille élémentaire du cristal et $2 W_{\mathbf{K}}$, l'énergie de formation d'un double décrochement.

Pour des températures largement supérieures à celle du pic de Bordoni, Mercier envisage que le mouvement des boucles de dislocations de longueur supérieure à $l_{\mathrm{c}}$ soit bien décrit par la théorie de la corde vibrante Le frottement intérieur et le défaut de module dans le modèle des décrochements géométriques ou de la corde vibrante sont donnés formellement par les mêmes équations, mais les constantes qui interviennent sont différentes et une dislocation de longueur libre $l$ engendrerait un frottement intérieur plus important dans le modèle des décrochements géométriques que dans celui de la corde vibrante. L'effet d'une irradiation à une température supérieure à celle du pic de Bordoni diminuant $l$ modifie la distribution des dislocations vibrant suivant le modèle des décrochements géométriques et de celles décrites par le modèle de la corde vibrante. Mercier montre ainsi qu'un peaking effect pourrait apparaitre sous les conditions suivantes :

- la température doit être supérieure à celle du pic de Bordoni,

- la quantité $\omega B / K$ due aux décrochements géométriques doit être de l'ordre de 1 ,

- les contraintes internes de l'échantillon doivent être telles que la longueur libre des dislocations soit de l'ordre de $l_{\mathrm{c}}$. Si elle est trop faible, le peaking effect n'apparaîtra pas.

La figure 1 montre que dans l'argent, aux fréquences auxquelles nous travaillons, le maximum du pic de Bordoni se trouve vers $75 \mathrm{~K}$. Sur la figure 9, nous observons la variation du peaking effect avec la température d'irradiation. On constate que ce phénomène n'est pas observé pour des températures inférieures à $80 \mathrm{~K}$, qu'il apparaît à cette même température et qu'il se développe en amplitude jusqu'au moins $300 \mathrm{~K}$. Cette variation thermique est tout à fait compatible avec l'hypothèse de Mercier.

Il est intéressant de noter les valeurs numériques de $\omega B / K$ au cours de ces variations; elles sont de l'ordre de 0,1 .
Les échantillons d'argent que nous avons étudiés sont préalablement recuits à haute température, et après montage dans le cryostat, sont recuits in situ afin d'éliminer toute trace d'écrouissage; les contraintes internes sont donc faibles ce qui est la condition pour observer le rôle des contraintes externes appliquées à l'échantillon. On observe sur la figure 7 qu'à $170 \mathrm{~K}$, le peaking effect augmente avec la déformation appliquée. Le rapport des contraintes étudiées ici n'est pas très élevé et il faudrait pouvoir descendre à des valeurs plus faibles de sollicitation pour observer comme Lauzier et al. [3] dans le cuivre une disparition du peaking effect lorsque la déformation devient très faible.

Pour les deux échantillons étudiés sur les figures 9 et 11 , les longueurs libres des dislocations sont certainement sensiblement différentes comme Stadelmann et al. [20] l'ont observé dans des échantillons d'argent de puretés comparables, ce qui peut expliquer que le peaking effect ne soit pas quantitativement identique dans les deux cas.

En résumé, dans l'argent, le comportement du frottement intérieur et du module élastique que nous avons observé au cours d'irradiations à diverses températures s'interpréterait donc qualitativement assez bien par le modèle de Mercier du peaking effect ; dans le cas du cuivre au contraire, Lauzier (à paraître) montre que certaines observations expérimentales ne concordent pas avec cette interprétation.

5. Conclusion. - Les expériences que nous avons réalisées dans l'argent apportent une réponse très claire sur la nature du premier stade d'ancrage des dislocations observé à basse température lors de recuits isochrones effectués après bombardement électronique à $20 \mathrm{~K}$. Ce stade apparaît entre 25 et $50 \mathrm{~K}$ et correspond très bien au stade $\mathrm{I}_{\mathrm{E}}$ de revenu de la résistivité associé à la migration à longue distance de l'interstitiel libre.

Lorsque les irradiations sont effectuées à des températures plus élevées, on observe le phénomène de peaking effect que nous avons caractérisé en faisant varier les paramètres de la température d'irradiation, du flux instantané de particules incidentes, de la déformation de mesure et de la nature de l'échantillon. Les résultats ont été confrontés aux théories existantes et dans le cas de l'argent ; celle de Mercier [16] qui tient compte du mouvement des dislocations par l'intermédiaire des décrochements géométriques et des doubles décrochements thermiques semble être la seule qui, pour l'instant, interprète à la fois les variations thermiques et avec la déformation du phénomène que nous observons.

Remerciements. - Nous sommes reconnaissants à Monsieur J. Lauzier pour les fructueuses discussions que nous avons pu avoir avec lui et à Messieurs D. Duclos et A. Séon pour leur aide technique, ainsi qu'au personnel du Service des Basses Températures. 


\section{Bibliographie}

[1] Thompson, D. O., Pare, V. K., Phys. Acoust. III A (1966) 293.

[2] Sosin, A., KeEFer, D. W., Adv. Mat. Res. 2 (1968) 159.

[3] Lauzier, J., Minier, C., Seiffert, S. L., Philos. Mag. 31 (1975) 893.

[4] KoenleR, J. S., Imperfections in Nearly Perfect Crystals, W. Schockley et al. Eds. (Wiley, New York) 1952, p. 197.

[5] Granato, A. and Lucke, K., J. Appl. Phys. 27 (1956) 582, 789.

[6] Seeger, A., Schiller, P., Acta Met. 10 (1962) 348.

[7] Alefeld, G., J. Appl. Phys. 36 (1965) 2642.

[8] Van Konynenburg, R. A. and Guinan, M. W., Fundamental Aspects of Radiation Damage in Metals, edited by M. T. Robinson and F. W. Young, Jr. ERDA Report CONF 751006 vol. II, p. 970.

[9] Wiffen, F. W., Snead, C. L., Jr. and Kauffman, J. W., Phys. Status Solidi 32 (1969) 459.

[10] Rizk, P., Vajda, P., Maury, F., Lucasson, A. and Lucasson, P., J. Appl. Phys. 48 (1977) 481.
[11] Simpson, H. M., Sosin, A., Phys. Rev. B 5 (1972) 1382.

[12] Sosin, A., Internal Friction and Ultrasonic Attenuation in Crystalline Solids (Springer Verlag, Berlin) 2 (1975) 109.

[13] Lenz, D., LuCKe, K., Internal Friction and Ultrasonic Attenuation in Crystalline Solids (Springer Verlag, Berlin) 2 (1975) 48.

[14] SeEger, A., Internal Friction and Ultrasonic Attenuation in Crystalline Solids (Springer Verlag, Berlin) 2 (1975) 232.

[15] Granato, A. V., Fundamental Aspects of Radiation Damage in Metals, edited by M. T. Robinson and F. W. Young, Jr., ERDA Report CONF 751006, vol. II, p. 932.

[16] Soulie, J. C., Jodeau, J. P., Depierre, Y., Minier, C., Radiat. Eff. 11 (1971) 221.

[17] Thompson, D. O., Buck, O., Huntington, H. B. and Barnes, P. S., J. Appl. Phys. 38 (1967) 3057.

[18] Simpson, H. M. and Sosin, A., Phys. Rev. B 16 (1977) 1489.

[19] Pare, V. K., J. Appl. Phys. 32 (1961) 332.

[20] Stadelmann, P. and Benoit, W., Scr. Met. 11 (1977) 645. 